Invited oral presentation at

International Conference on Metallurgical Coatings and Thin Films '2004

San Diego, California , 19-23 April 2004

Text to appear in Surface Coatings Technology

\title{
CVD and CVI of pyrocarbon from various precursors
}

Gerard L. Vignoles, Francis Langlais, Cédric Descamps, Arnaud Mouchon, Hélène Le Poche, Nicolas Reuge and Nathalie Bertrand

Laboratoire des Composites ThermoStructuraux (LCTS)

3, Allée La Boëtie- Domaine Universitaire

F 33600 PESSAC, France

\begin{abstract}
The control of pyrocarbon Chemical Vapor Infiltration (CVI) is a key issue in the processing of high-performance $\mathrm{C} / \mathrm{C}$ composites with applications in aerospace parts and braking technology. For years, the precise investigation of deposition kinetics and pyrocarbon nanometer-scale anisotropy has been rehearsed in CVD and several variants of CVI with various pore sizes, and using mostly propane, propylene, and methane as source precursors.

A literature survey and the analysis of recent experimental data have helped to understand better the role of gas-phase intermediate species in the various nanotextural transitions ; a coherent modeling frame, suitable for propane, propylene, and methane - the latter having a neatly lower reactivity - has been set up and tested against experimental results from independent teams. The relation between nanotexture and processing conditions are then explained.
\end{abstract}




\section{INTRODUCTION}

Carbon/carbon $(\mathrm{C} / \mathrm{C})$ composite materials are used in high-temperature applications, such as rocket nozzles, heat shields for atmospheric reentry, airplane and F-1 braking, and furnace components. The highest thermal and mechanical quality is obtained for CVIprocessed pieces. CVI [1] means Chemical Vapor Infiltration, a process derived from CVD in which a preform made of carbon fibers is densified by a pyrocarbon (pyC) deposit originated in the cracking of gaseous hydrocarbons, usually at high temperatures (ca. 1000-1500 K) and low pressures $(\mathrm{ca} .1-50 \mathrm{kPa}$ ). This process allows the fabrication of complex pieces without damaging the carbon fibres; moreover, it is possible to deposit very anisotropic pyrocarbons.

Hydrocarbon pyrolysis is known to lead to various nanotextural forms of pyrocarbon in the context of CVD and/or CVI [2,3], ranging from nearly isotropic to highly anisotropic (i.e. close to graphite structure). The CVD- and CVI-made anisotropic nanotextures are termed "Laminar" pyrocarbons. Among them, two varieties have been recognized, and have been called "Rough Laminar" (RL) and "Smooth Laminar" (SL) because of their appearances when imaged by Polarized Light Optical Microscopy (PLOM). They differ by their degree of structural anisotropy, and have distinct mechanical and optical properties. Moreover, only the $\mathrm{RL}$ form is graphitizable by a high-temperature post-treatment [3,4]. A key issue in pyC CVD/CVI is the control of the deposit nanotexture during processing. It has been shown [5-7] that processing parameters such as temperature $T$, pressure $P$, and composition ratios are important for this, as well as residence time $t_{\mathrm{s}}$ and surface-to-volume ratio $S_{\mathrm{V}}$ [8]. The existence diagrams in processing parameter space of the various nanotextures has been subject to strong controversies, because of the large differences between experimental setups and of the versatility of the pyrolysis-deposition chemical system. Indeed, it is well known that hydrocarbon pyrolysis rapidly leads to hundreds of distinct molecular or radical species, and one the key issues of pyrocarbon nanotexture control is the identification of the most 
important species and sub-mechanisms that may be associated to a given nanotexture. The nature of the starting species and the precise physico-chemical conditions may alter strongly the gas-phase composition and reactivity. In the following, we will not use the traditional "RL-SL" notation, but rather distinguish between High-Anisotropy (HA) and LowAnisotropy (LA) pyrocarbon forms, since it has been found that some highly anisotropic pyrocarbons do not display a rough Maltese Cross PLOM feature [8]. Medium anisotropy will be denoted MA. The extinction angles for LA are $A_{e} \leq 12^{\circ}$; for $\mathrm{HA}: \mathrm{A}_{\mathrm{e}} \geq 18^{\circ}$, and MA in between [9].

One of the most confusing points is that CVD observations and models are not readily transposable into CVI models, for at least three reasons. First, because of the high $S_{\mathrm{V}}$ values, heterogeneous chemistry plays a much more important role in CVI in contrast to CVD [10]. For instance, if saturation adsorption may occur in CVD, this is much less possible in CVI, at least far from the porous medium surface. Also, some pyrolysis sub-mechanisms which exist in the gas phase may be completely overridden by heterogeneous ones, leading to a very different apparent behavior of the gas phase. Second, depletion effects are to be expected in narrow pores, due to transport limitations. This latter point has been thoroughly investigated in numerous modeling works, dealing either with isothermal-isobaric CVI (I-CVI) [12-14], forced-CVI (F-CVI) [15], thermal-gradient-CVI (TG-CVI) either with microwave or radiofrequency heating (see a review in [16]). Third, even with very simple kinetic models, the depletion effects that are to be expected outside the perform may be very important in CVI cases with very high $S_{\mathrm{V}}$ values [17]. The preform is much more reactive than a plane substrate, so the diffusional boundary layer which surrounds it is much more marked, and may easily reach the outer walls [18]. Taking these facts into account is only feasible in a global modelling frame where both the preform and the surrounding free-medium are represented. 
We will try to depict the current modeling efforts towards the understanding of pyrocarbon CVD/CVI. They start from three kinds of experiments : CVD on a plain substrate, with a very low $S_{\mathrm{V}}$ ratio ; CVI with a fibrous preform displaying very high $S_{\mathrm{V}}$ values ; and intermediate situations that may be called "weakly-coupling CVI" (wc-CVI).

CVD has the strong advantage of being much simpler than CVI, since $i$ ) it does not involve gas transport in porous media, and ii) there is a negligible feedback effect from heterogeneous reactions in a wide range of conditions - that is, they do not alter significantly the gas-phase composition, and thus the homogeneous reactions. An identification of the chemical processes is thus apparently easier to perform in a well-controlled reactor, for which all of the above mentioned processing parameters may be monitored. On the other hand, CVI experiments are close to the desired applications and give a larger role to the heterogeneous reactions, that could be poorly characterized in some CVD situations. However, the feedback effect depletes the apparent concentrations of precursor gases at pore mouths, thus yielding a bias in the results and possible errors in the kinetic rate identifications. This is why "weaklycoupling CVI", in which $S_{\mathrm{V}}$ is high enough to enhance heterogeneous mechanisms but low enough to avoid a strong feedback, is of special interest in trying to understand the whole reaction sequence.

In the first part of this paper, we will briefly present the main experimental results for propane and methane as precursors. New experimental data for propane indicate that deposition of a HA form of laminar pyrocarbon is possible at very low residence times.

Then, an extension of the current models for propane will be presented. Another characteristic intermediate will be identified, with an associated tentative deposition mechanism. Then, the model will be tested with other gas-phase precursors, namely methane, for which numerous experimental data are available, as well as propene. 


\section{PYROCARBON DEPOSITION : RATES AND NANOTEXTURE}

Many experimental studies have shown in the past the importance of processing parameters on the pyC deposition rates and nanotexture, in various physico-chemical conditions and reactor configurations, either in CVD (plain substrate, low $S_{\mathrm{V}}$ ), or in CVI (porous substrate, high $S_{\mathrm{V}}$ ) [19-36]. Most of them have tried to identify some "ultimate precursor" of pyC, either light, aliphatic species, or heavy aromatic compounds such as PAHs (Polycyclic Aromatic Hydrocarbons) and polyynes. It has been proved, from mass spectroscopy $[28,32]$, gas chromatography $[33,34]$ and FT-IR $[35,36]$ measurements of the gas-phase composition, that the hydrocarbon pyrolysis follows a long chain of homogeneous reactions, in a so-called "maturation" process : $i$ ) precursor decomposition, $i i$ ) recombination of the first products into other species among which unsaturated species and resonancestabilized free radical species (RSFRs), $i i i$ ) growth of heavier molecules with a varying degree of unsaturation or aromaticity, among which PAHs (Polycyclic Aromatic Hydrocarbons), and, more hypothetically, polyynes.

The pyrocarbon deposits may originate from both light and heavy hydrocarbons, probably with distinct mechanisms : in the case of heavy precursors, a condensation-like mechanism has been proposed [37], while for light species, a lateral growth mechanism, close to a radical-based polymerization reaction scheme, has been discussed $[38,39]$. It has to be expected that the overall reaction rate dependence on temperature should vary with temperature (since the activation energies may be different), on the total pressure and residence time (since the relative amounts of various key species may vary), and on $S_{\mathrm{V}}$ (since the heterogeneous to homogeneous reaction ratio varies). Indeed, all those parameters may toggle the system between various dominant mechanisms.

Using pure propane as a precursor, the reaction rate in CVD conditions and the deposit anisotropy in wc-CVI conditions have been recorded in the same experimental apparatus [34$36,40-42]$. 
In early results, the nanotexture was found to undergo a neat transition from low (LA) to a high (HA) anisotropy form and back to LA when the residence time or the temperature increases $[40,41]$. The overall CVD reaction rate has been found to increase neatly when HA pyC is obtained - in constrast with LA pyC - in wc-CVI conditions. However, new experimental data at very low residence times and different pressures [42], in the case of propane as a precursor, have evidenced the possibility of depositing highly anisotropic pyC. Fig. 1a summarizes the CVD reaction rates and wc-CVI nanotextures for pure propane at $950^{\circ} \mathrm{C}$ and pressures ranging from 0.5 to $5 \mathrm{kPa}:$ a new, smaller reaction rate maximum appears around $t_{s} \sim 0.1$. The associated nanotexture is LA in CVD conditions, but is HA at some distance inside the pores in wc-CVI conditions. Also, at the lowest studied pressure, the (second) LA HA transition previously reported disappears.

In full CVI conditions, an HA LA HA transition sequence has been reported [34]. This confirms the fact that two distinct HA pyC deposition mechanisms are present, acting at distinct maturation conditions.

Thus, the complete CVI sequence when residence time increases is now (L,M)A HA LA HA LA. Propane pressure affects this scheme : when it decreases enough, the sequence is now (L,M)A HA LA, that is, the second transition towards HA disappears.

These facts are to be put in parallel with other experimental results obtained in CVI conditions with methane as a precursor [43], where it is shown that as methane pressure increases, the anisotropy of pyC follows the sequence MA HA LA, and the reaction rate (indeed, the bulk density after a given infiltration time) displays a maximum when HA pyC is deposited. Also, the possibility of inside-out densification [44] arises from the existence of such a maximum. 


\section{PAST MODELS : A SHORT REVIEW}

From a chemical kinetic point of view, the modeling efforts concerning pyC deposition have been devoted primarily to the study of the maturation phenomenon, that is, to hydrocarbon pyrolysis. The gas-phase reaction steps have been successfully modeled using comprehensive [45,46] or semi-detailed [47] mechanisms. They have been also followed by the incorporation of heterogeneous reactions, but with considerably less details. On the other hand, models taking transport into account feature much less detailed chemistry, for obvious reasons of computational limitations. Thus, a coherent modeling of pyC CVD/CVI that accounts for the pyC nanostructure has to reduce the information arising from detailed chemistry.

In the case of propane decomposition at $P=2 \mathrm{kPa}, \mathrm{T}=900^{\circ} \mathrm{C}-1200^{\circ} \mathrm{C}$, and moderate to large residence times, in a tubular reactor and CVD conditions, a kinetic modeling study based on a detailed gas-phase mechanism has confirmed the maturation phenomenon and its importance on one of the LA/HA transitions (from LA to HA) when residence time and/or temperature increases $[45,46]$. It appeared that only the heaviest species in the detailed model could be associated to HA pyrocarbon growth, while many lighter ones were related to LA (see figures 1a and c). Later on, a complete CVD-to-CVI procedure has been developed $[48,18]$.

First, a CVD simplified model has been produced from the detailed pyrolysis study [46]; then, experimental data has been used for the identification of some rate constants and activation energies ; next, a 1D CVD solver has been used for the determination of the remaining parameter values. The "PAH incubation" phenomenon has been accounted for by turning the last gas-phase reaction non-linear, i.e. considering that HA pyC deposition may occur only if the partial pressure of the heaviest species exceeds a given threshold. It has turned out that various parameter sets could be chosen, with a similar correlation quality. One of the successes of this CVD model is that the last HA LA transition has also been 
accounted for, since the presence of the HA deposition reaction implies an appreciable depletion of the heaviest species at very large residence times. In a second phase, model-pore infiltrations have been performed. The model porous medium was such that its effective transport coefficients were precisely known during infiltration, and it has been checked that the $S_{\mathrm{V}}$ ratio was low enough to ensure that the feedback of the preform reactivity on the surrounding gas-phase was negligible (wc-CVI conditions), thus allowing to relate rather directly heterogeneous reactions to the species partial pressures resulting from the gas-phase decomposition computations. The same reactor as for CVD has been used.

One-dimensional CVI computations have been performed in order to reproduce the deposit thickness profiles. This has only been possible introducing some new hypotheses concerning the intermediate species groups and related individual deposition rates, thus altering somewhat the initial CVD model. The propane-CVI model obtained is summarized in fig. 2 and table 1. It has managed to reproduce successfully either CVD or CVI experiments using propane at moderate to large residence times. This model has also been injected into a coupled solver featuring both free-medium and porous medium described in [17], and it has been checked that the backwards influence of the in-pore deposition on the free medium was negligible. Such a model allowed to understand how the second HA form of pyC appears, but failed completely to explain the onset of the first HA pyC deposition (at short residence times).

In the case of pyC deposition from methane, the detailed models of pyrolysis show that the characteristic times for pyrolysis to begin are considerably higher than those for propane, due to the exceptional stability of this molecule (no possibility of C-C bond breaking). Once the pyrolysis has begun, attention is paid mostly to acetylene and benzene as key intermediates for the growth of pyC at low residence times, and PAHs for large residence times. This is in complete coherence with the propane-based chemical scheme. 
Recent simulation studies [49,50] with parameter identification based on CVD [28] and CVI [30,23] experiments tend to confirm the idea that $\boldsymbol{B 1}, \boldsymbol{B 2}$, and $\boldsymbol{C}$ groups (see table 1) also act in the case of deposition from methane. The main difference is now that $\mathrm{CH}_{4}$ should be considered both as a starting point for the mechanism and as a $\boldsymbol{B} \boldsymbol{O}$ group species; the formal scheme presented for propane does not have to change, only the $\mathrm{H}_{2}$ production stoichiometries have to be revised, as well as the rate constant \#1, which is considerably lower.

However, it has been argued that HA deposition from methane arises principally when $\boldsymbol{B}$ group species are present, since, at the considered experimental conditions (higher $P$ and $T$ ), homogeneous nucleation of soot predominates over the formation of HA pyC. To explain this, a "particle-filler" model has been proposed [43], asserting that the degree of anisotropy is susceptible to increase when a particular $\mathrm{C}_{2} \mathrm{H}_{2} / \mathrm{C}_{6} \mathrm{H}_{6}$ ratio is attained, and this should occur at low residence times. This model states that very anisotropic pyrocarbon may be deposited by lateral growth of graphene planes, for which the lowest quantity of defaults (e.g. C5 or C7rings, helicoidal structures) is attained when $\mathrm{C}_{2} \mathrm{H}_{2}$ acts as a "filler" between $\mathrm{C}_{6} \mathrm{H}_{6}$ "particles".

\section{CORRELATION OF EARLY HA pyC DEPOSITION WITH GAS PHASE}

In order to understand the whole chemical scheme, the simulations with detailed gasphase pyrolysis mechanism have been extended to very short residence times in the case of propane, and to the case of methane and propene decomposition. Either 0D (batch reactor) or 1D (tubular reactor with radial effects neglected) computations have been performed, and heterogeneous reactions have been neglected, since the results will be compared to experimental data acquired at low $S_{\mathrm{V}}$ ratios.

A preceding 0D study [51] had shown that $\mathrm{CH}_{4}$ decomposition has a neatly distinct behavior with respect to $\mathrm{C}_{3} \mathrm{H}_{8}$ and $\mathrm{C}_{3} \mathrm{H}_{6}$. Indeed, the build-up time for intermediate species such as $\mathrm{C}_{2} \mathrm{H}_{2}$ and $\mathrm{C}_{6} \mathrm{H}_{6}$ is shifted towards higher ts and temperature values. This confirms 
experimental knowledge, and arises from the high activation energy for $\mathrm{C}-\mathrm{H}$ bond breaking, crucial in the first step of methane decomposition chemistry. Additionally, this first step is a "third-body enhanced" reaction :

$$
\mathrm{CH}_{4}+\mathrm{M} \quad \mathrm{H}+\mathrm{CH}_{3}+\mathrm{M}
$$

which gives an important role either to total pressure or to $S_{\mathrm{V}}$ (M being respectively any gaseous species or the surface).This special feature of $\mathrm{CH}_{4}$ has been successfully used to undertake inside-out isothermal CVI [44]. The propene case seems very similar to propane, since in the considered conditions, the main decomposition route for $\mathrm{C}_{3} \mathrm{H}_{8}$ is a dehydrogenation into $\mathrm{C}_{3} \mathrm{H}_{6}$.

The same chemical model has been reused for new $1 \mathrm{D}$ calculations using either $\mathrm{C}_{3} \mathrm{H}_{8}$ or $\mathrm{CH}_{4}$ as pure precursors, in a hot-wall tubular reactor with $S_{\mathrm{V}}$ ratio $=118 \mathrm{~m}^{-1}$. In the propane case, the new experimental facts have been put in relation with the early build-up of $\mathrm{C} 3$ species. Figures 1a-c are a comparison of experimental CVD growth rates and wc-CVI extinction angles with scaled partial pressures and partial pressure ratios, computed at the reactor outlet. The hot-zone temperature was $1223 \mathrm{~K}$ and the pressure $0.5 \mathrm{kPa}$ (fig. 1 ) and 5 $k P a$ (not shown). The early $\mathrm{HA}$ pyC deposition is possibly related either to the $\mathrm{C}_{6} \mathrm{H}_{6} / \mathrm{C}_{2} \mathrm{H}_{2}$ ratio, or to small dehydrogenated species such as $\mathrm{C}_{3} \mathrm{H}_{4}$ or $\mathrm{C}_{3} \mathrm{H}_{5}$. The $\mathrm{C}_{6} \mathrm{H}_{6} / \mathrm{C}_{2} \mathrm{H}_{2}$ optimal ratio for HA pyC growth seems to be slightly different at the two considered pressures $(0.04$ at $5 \mathrm{kPa}$ and 0.06 at $0.5 \mathrm{kPa}$ ) and in any case it is very low.

In the case of methane, $1 \mathrm{D}$ computations were carried out at $1373 \mathrm{~K}$ with total pressure $100 \mathrm{kPa}$, residence time in hot zone $t_{\mathrm{s}}=0.33 \mathrm{~s}, S_{\mathrm{V}}=7,6 \mathrm{~m}^{-1}$, and methane partial pressures ranging between 5 and $35 \mathrm{kPa}$, argon being used as a dilution species. Previous CVD [33,52] and CVI [53] experimental results have been reported, and will be compared both to propane results and to the numerical results of this study, summarized at figure 3 . Also, a $1 \mathrm{D}$ numerical study of the influence of residence time at inlet $P\left(\mathrm{CH}_{4}\right)=10 \mathrm{kPa}$ is shown at fig. 4. 
The first striking fact is that the reported evolutions of experimental CVI pyC anisotropy are very similar between methane as a function of pressure and propane as a function of residence time (see figs. 1a and 3a). Indeed, the sequence HA LA HA is also observed. Note that in the methane case, the anisotropy in CVD experiments has not been determined simultaneously with the growth rates, and that it has not been studied in CVI or wc-CVI as a function of residence time.

The measured behavior of the main C2 gas phase species [33] is successfully reproduced, as well as the $\mathrm{C}_{6} \mathrm{H}_{6} / \mathrm{C}_{2} \mathrm{H}_{2}$ evolution. It may be seen that the "critical ratio" has a different computed value in the methane case (computed around 0.15 , experimentally lies around 0.25 ) compared to the propane case (computed around 0.05). Again, $\mathrm{C}_{2} \mathrm{H}_{2}$ and $\mathrm{C}_{6} \mathrm{H}_{6}$ display very similar evolutions.

The computed evolutions of C2, C3 and C6 species exhibit a classical ordering sequence of the relative maxima :

$$
\begin{array}{lllll}
\mathrm{CH}_{4} & \mathrm{C}_{2} \mathrm{H}_{6} & \mathrm{C}_{2} \mathrm{H}_{4} & \mathrm{C}_{2} \mathrm{H}_{2} & \mathrm{C}_{6} \mathrm{H}_{6}
\end{array}
$$

But it has to be noted that C3 species also appear very early, although with a very low absolute amount :

$$
\begin{gathered}
\mathrm{CH}_{4} \mathrm{C}_{2} \mathrm{H}_{6} \quad \mathrm{C}_{2} \mathrm{H}_{4} \quad \mathrm{C}_{2} \mathrm{H}_{2} \\
\longrightarrow \mathrm{C}_{3} \mathrm{H}_{5} \longrightarrow \mathrm{C}_{3} \mathrm{H}_{4}
\end{gathered}
$$

So, again, the C3 intermediate species can not be discarded as potential precursors for early HA deposition.

\section{GLOBAL MODEL FOR pyC DEPOSITION FROM VARIOUS PRECURSORS}

Setting up a global model of pyC deposition should take into account the gas-phase maturation (i.e. the onset of various species by bond breaking and recombination), and deposition mechanisms originating in various precursors, since the non-monotonous anisotropy evolution clearly suggests a variety of heterogeneous reactions. Concerning 
homogeneous reactions, it seems that the propane and methane cases are similar, at the noticeable exception of the initiation step, which is much slower for methane, and of the relative amounts of C3 species, which are also lower for methane.

First, the deposition of late HA is clearly related to high molecular weight species such as PAHs. This arises both from experimental [34-36] and computational $[46,30,18]$ facts. A contrario, the intermediate LA form of pyC seems to be correlated to species with lower molecular weights. A previous work [48] points at $\mathrm{C}_{2} \mathrm{H}_{2}$ and $\mathrm{C}_{6} \mathrm{H}_{6}$ as possible sources acting in parallel, because of neat slope breaks in CVI deposition profiles.

Then, the case of the early HA pyC deposition has to be dealt with. Again, species with low molecular weights have to be considered ; the study of evolutions with residence time strongly indicate that the key species have to be early products of pyrolysis (first- or second-generation products). Two main theories may be discussed : the "particle-filler theory" and the " $\mathrm{C} 3$ route theory".

The "particle-filler theory" relies on the topological argument that C5-ring formation is prevented when a suitable $\mathrm{C}_{6} \mathrm{H}_{6} / \mathrm{C}_{2} \mathrm{H}_{2}$ ratio is attained : in such a case, the $\mathrm{C}_{2} \mathrm{H}_{2}$ species will exactly "fill" the holes left out by the $\mathrm{C}_{6} \mathrm{H}_{6}$ "particles". This point is critical for the lateral growth of zigzag-shaped graphene edges, for which direct addition of either pure $\mathrm{C}_{2} \mathrm{H}_{2}$ or pure $\mathrm{C}_{6} \mathrm{H}_{6}$ would yield 5-membered rings, which cause the presence of local curvatures and thus decrease the amount of pyC anisotropy. However, it is difficult to imagine the precise bi- or multi-molecular mechanism through which $\mathrm{C}_{2} \mathrm{H}_{2}$ and $\mathrm{C}_{6} \mathrm{H}_{6}$ would yield perfectly matching additions. The present numerical results also give two informations in the propane and the methane case : first, $\mathrm{C}_{2} \mathrm{H}_{2}$ and $\mathrm{C}_{6} \mathrm{H}_{6}$ have a very similar evolution, even though $\mathrm{C}_{6} \mathrm{H}_{6}$ is formed later; second, the $\mathrm{C}_{6} \mathrm{H}_{6} / \mathrm{C}_{2} \mathrm{H}_{2}$ ratios correlated to early $\mathrm{HA}$ deposition are neatly different in the two cases. There is no obvious explanation for this latter fact.

On the other hand, from the results of the preceding sections, it appears that some $\mathrm{C} 3$ species could be a possible alternative explanation to the growth of early RL pyC. Indeed, if 
one considers the approach of boat-like or zigzag-like lateral growth sites of graphene layers by a $\mathrm{C} 3$ species, it is always possible to produce 6 -membered rings, as illustrated at figure 5 , even though C5-ring formation is not excluded. This is a simple argument for the growth of highly anisotropic pyC. Taking into account the early presence of C3 intermediates in all studied conditions, we retain the "C3-route" as a hypothesis for the global mechanism.

Summarizing all exposed arguments, it is possible to sketch a global frame for pyC deposition either from methane or from propane. Figs. $6 a$ and $b$ are qualitative schemes of the propane-based and methane-based mechanisms, from which the similarities and differences are put forward.

\section{SUMMARY AND CONCLUSIONS}

Pyrocarbon CVD and CVI modeling have been the object of studies since many decades and is still a topic of actuality, because of the complexity of the involved chemistry, and of its subtle entanglement with transport phenomena. The question of understanding and modeling pyrocarbon deposition from various precursors in CVD and CVI has been addressed through the examination of numerous experimental results, mainly in the case of either propane or methane as single precursors, and new 1D numerical simulations that can be directly related at least to CVD and to "Wc-CVI" (weak-coupling CVI) experiments. The growth rates and degree of pyrocarbon anisotropy have been correlated to gas-phase species appearing during maturation. The results have been summarized into a qualitative global scheme which is valid for both precursors, although marked differences exist in the system behavior. It appears that the main particularity of methane is related to its very slow $\mathrm{C}-\mathrm{H}$ bond breaking step, and that the rest of the gas-phase chemistry is rather similar to the routes followed by other hydrocarbons. The pyrocarbon deposition follows at least three distinct heterogeneous mechanisms, the relative importance of which depends on residence time, $S_{\mathrm{V}}$

ratio, and other control parameters. They rely on three distinct intermediate species pools. The 
non-monotonous evolution of CVI pyC anisotropy with control parameters is explained using these elementary routes.

There remains much complementary work in order to strengthen the qualitative scheme that has been constructed. Experimentally, more gas-phase characterization studies would help ensure the precise correlation of the intermediates with deposition mechanisms. New encouraging results are expected from e.g. molecular-beam mass spectrometry experiments [29]. On the level of modeling, there clearly remains to identify with more precision heterogeneous reaction step constants, and to validate the mechanism in more complicated $2 \mathrm{D}$ or 3D situations, which are closer to the experimental and industrial reality.

\section{ACKNOWLEDGEMENTS}

The authors wish to thank Snecma Propulsion Solide for financial support through PhD grants to C.D., N.R., H.L.P. and A.M., and Conseil Régional d'Aquitaine for financial support.

The authors also acknowledge fruitful discussion with L. Vandenbulcke (CNRS Orléans) and R. Fournet (ENSIC Nancy) and P.-M. Marquaire (CNRS, Nancy).

\section{LIST OF REFERENCES}

1. R. Naslain and F. Langlais, Mat. Sci. Res. 20 (1986) 145

2. J. C. Bokros, Chemistry and Physics of Carbon 5 (1969) 1

3. P. Loll, P. Delhaès, A. Pacault, A. Pierre, Carbon 15 (1977) 383

4. A. Oberlin, Carbon 22 (1984) 521

5. R.J. Diefendorf, in J. W. Mitchell, R. C. de Vries, and P. Cannon (eds.) Reactivity of solids, Wiley \& sons, New York (1969)

6. P. Lieberman and H.O. Pierson, Carbon 12 (1974) 233

7. H.O. Pierson and P. Lieberman, Carbon 13 (1975)159

8. X. Bourrat, A. Fillion, R. Naslain, G. Chollon and M. Brendlé, Carbon 40 (2002) 2931 
9. B. Reznik and K. J. Hüttinger, Carbon 40 (2002) 617

10. A. Becker and K. J. Hüttinger, Carbon 36 (1998) 225

11. K. J. Huttinger, Adv. Mater. - CVD 4 (1998) 151

12. J. Y. Ofori and S. V. Sotirchos, AIChE J. 42 (1996) 2828

13. S. K. Griffiths and R. H. Nilson, J. Electrochem. Soc. 145 (1998) 1263

14. P. McAllister and E. E. Wolf, AIChE J. 39 (1993)1196

15. T. L. Starr and A. W. Smith, Mat. Res. Soc. Symp. Proc. 168 (1990) 55

16. D. Leutard, G. L. Vignoles, F. Lamouroux and B. Bernard, J. Mater. Synth. and Proc. 9 (2002) 259

17. G. L. Vignoles, C. Descamps and N. Reuge, J. Phys. IV France 10 (2000) Pr2-9

18. N. Reuge, PhD thesis, Université Bordeaux 1, nº 2533 (2002)

19. K.M. Sundaram and G.F. Froment, Chem. Eng. Sci. 34 (1979) 635

20. R. Zou, Q. Lou, H. Liu and F. Niu, Ind. Eng. Chem. Res. 26 (1987) 2528

21. L.F. Albright and J.C. Marek, Ind. Eng. Chem. Res. 27 (1988) 755

22. D.B. Murphy and R.W. Carroll, Carbon 30 (1992) 47

23. S. Bammidipati, G.D. Stewart, J. R. Elliot Jr., S.A. Gokoglu, and M.J. Purdy, AIChE Journal 42 (1996) 3123

24. C.J. Chen and M.H. Back, Carbon 17 (1979) 175

25. J.Y. Lee, J.H. Je, and H.S. Kim, Carbon 21 (1983) 523

26. P. Lucas and A. Marchand, Carbon 28 (1990) 207.

27. P. Dupel, R. Pailler, X. Bourrat, and R. Naslain, J. Mater. Sci. 29 (1994) 1056

28. W. G. Zhang, and K.J. Hüttinger, J. Mater. Sci. 36 (2001) 3503

29. O. Aubry, PhD Thesis, University of Orleans (2002)

30. Z. Hu and K.J. Hüttinger, Carbon 39 (2001) 1023

31. H.S. Park, W.C. Choi, and K.S. Kim, J. Adv. Mater. 26 (1995) 34

32. F. Fau-Canillac, F. Carrere, A. Reynes, C. Vahlas and F. Maury, J. Phys IV France 5 (1995) C5-89

33. A. Becker and K.J. Hüttinger, Carbon 36, (1998) 177, 201, 213 and 225

34. J. Lavenac, PhD Thesis, Université Bordeaux I, nº2274 (2000) 
35. O. Féron, F. Langlais, R. Naslain, and J. Thebault, Carbon 37 (1999) 1343

36. O. Féron, F. Langlais, and R. Naslain, Carbon 37 (1999) 1355

37. M. Tesner in Proc. $7^{\text {th }}$ Symposium (Intl.) on Combustion, Butterworths, London, 1959, p. 546 ; R. J. Diefendorf, J. Chem. Phys 57 (1960) 815

38. M. Frenklach, in A. R. Burgess et al. (eds.), Proc. $26^{\text {th }}$ Symposium (Intl.) on Combustion, The Combustion Institute, Pittsburgh, 1996, p.2295

40. J. Lavenac, F. Langlais, O. Féron and R. Naslain, Compos. Sci. and Technol. 61 (2001) 339

41. J. Lavenac, F. Langlais, X. Bourrat and R. Naslain, J. Phys. IV France 11 (2001) Pr31013

42. H. Le Poche, PhD Thesis, University Bordeaux 1, $\mathrm{n}^{\circ} 2657$ (2003).

43. W. G. Zhang, Z. J. Hu and K. J. Hüttinger, Carbon 40 (2002) 2529

44. W. G. Zhang, and K.J. Hüttinger ECS Proceedings PV 2003-08 (2003) 549

45. C. Descamps, G. L. Vignoles, O. Féron, F. Langlais and J. Lavenac, J. Phys. IV France 11 (2001) Pr3-101

46. C. Descamps, G. L. Vignoles, O. Féron, F. Langlais and J. Lavenac, J. Electrochem. Soc. 148 (2001) C695

47. N. Birakayala and E. A. Evans, Carbon 40 (2002) 675

48. N. Reuge, G. L. Vignoles, H. Le Poche, and F. Langlais, Adv. Sci. Technol. 36 (2002) 259.

49. W. Benzinger and K. J. Hüttinger, Carbon 37 (1999) 941

50. W. G. Zhang, and K.J. Hüttinger, Compos. Sci. And Tech. 62 (2002) 1947

51. G. L. Vignoles, F. Langlais, N. Reuge, H. Le Poche, C. Descamps and A. Mouchon, ECS Proceedings PV 2003-08 (2003) 144.

52. V. de Pauw, B. Reznik, S. Kalhöfer, D. Gerthsen, Z. J. Hu and K. J. Hüttinger, Carbon $41(2003) 71$

53. M. Guellali, R. Oberacker, M. J. Hoffmann, W. G. Zhang and K. J. Hüttinger, Carbon $41(2003) 97$ 


\section{TABLE CAPTIONS}

Table 1. Summary of the ex-propane CVD/CVI chemical model, validated for moderate to high residence times [48]. 




Table 1. 


\section{FIGURE CAPTIONS}

Figure 1. Evolution of measured [42] pyrocarbon CVD deposition rate, CVI nanotexture, and computed gas-phase species, for $T=1223 \mathrm{~K}$ and pure propane as a precursor, as a function of residence time. a) CVD rate for $P=5 \mathrm{kPa}, 2 \mathrm{kPa}$, and $0.5 \mathrm{kPa}$, and CVI nanotexture for $P=0.5 \mathrm{kPa}$ and $5 \mathrm{kPa}$. b) Computed outlet relative partial pressures of $\mathrm{C}_{3} \mathrm{H}_{8}, \mathrm{CH}_{4}, \mathrm{C}_{2} \mathrm{H}_{4}, \mathrm{C}_{3} \mathrm{H}_{6}, \mathrm{C}_{3} \mathrm{H}_{5}, \mathrm{p}-\mathrm{C}_{3} \mathrm{H}_{4}, \mathrm{C}_{2} \mathrm{H}_{2}, \mathrm{C}_{6} \mathrm{H}_{6}$ and $\mathrm{C}_{10} \mathrm{H}_{8}$, at $P=0.5 \mathrm{kPa}$. c) Computed $\mathrm{C}_{6} \mathrm{H}_{6} / \mathrm{C}_{2} \mathrm{H}_{2}$ ratio, at $P=0.5 \mathrm{kPa}$. The shaded areas indicate residence times for which HA pyC is obtained at $5 \mathrm{kPa}$ (right) and all pressures (left).

Figure 2. Sketch of the ex-propane CVD/CVI chemical model, validated for moderate to high residence times [48]. See table 1 for numerical values.

Figure 3. Evolution of measured CVD deposition rate [33] and CVI nanotexture [53], and experimental [33] and computed species partial pressures, for $T=1373 \mathrm{~K}, t_{\mathrm{s}}=0.33$ $s$, total pressure $P=100 \mathrm{kPa}$, and methane/argon mixtures as feeding gas, as a function of inlet methane partial pressure. a) CVD deposition rate and CVI nanotexture. b) Measured and computed partial pressures of $\mathrm{C}_{2} \mathrm{H}_{2}, \mathrm{C}_{2} \mathrm{H}_{4}, \mathrm{C}_{2} \mathrm{H}_{6}$ and $\mathrm{C}_{6} \mathrm{H}_{6}$. c) Computed $\mathrm{C}_{6} \mathrm{H}_{6} / \mathrm{C}_{2} \mathrm{H}_{2}$ ratio.

Figure 4. Evolution of computed gas-phase partial pressures as a function of residence time for $\mathrm{CH}_{4} / \mathrm{Ar}$ mixtures as inlet gases in CVD conditions, $T=1373 \mathrm{~K}, P=100 \mathrm{kPa}$, inlet $P\left(\mathrm{CH}_{4}\right)=10 \mathrm{kPa}$.

Figure 5. Scheme of C6-ring formation from the addition of a C3-species on a "zigzag" or a "boat" graphene edge site.

Figure 6. Global qualitative scheme for pyrocarbon deposition and nanotexture. a) case of propane or propene as precursor. b) case of methane as a precursor. 
a)



b)
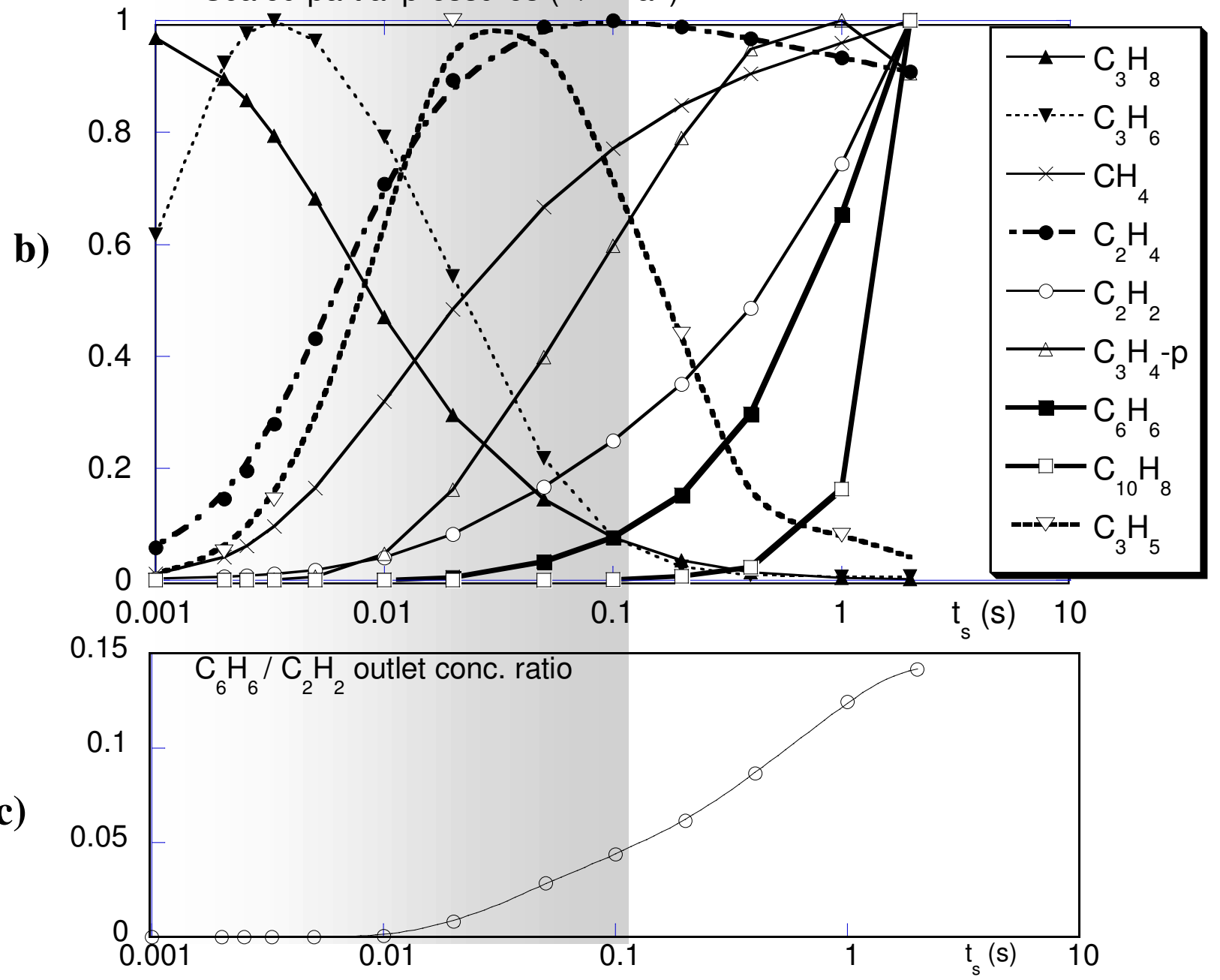

Figure 1. 
Gerard L. Vignoles, Francis Langlais, Cédric Descamps, Arnaud Mouchon,

Hélène Le Poche, Nicolas Reuge and Nathalie Bertrand

Figure 1. 




Fig. 2. 
Gerard L. Vignoles, Francis Langlais, Cédric Descamps, Arnaud Mouchon,

Hélène Le Poche, Nicolas Reuge and Nathalie Bertrand

Figure 2. 
a)
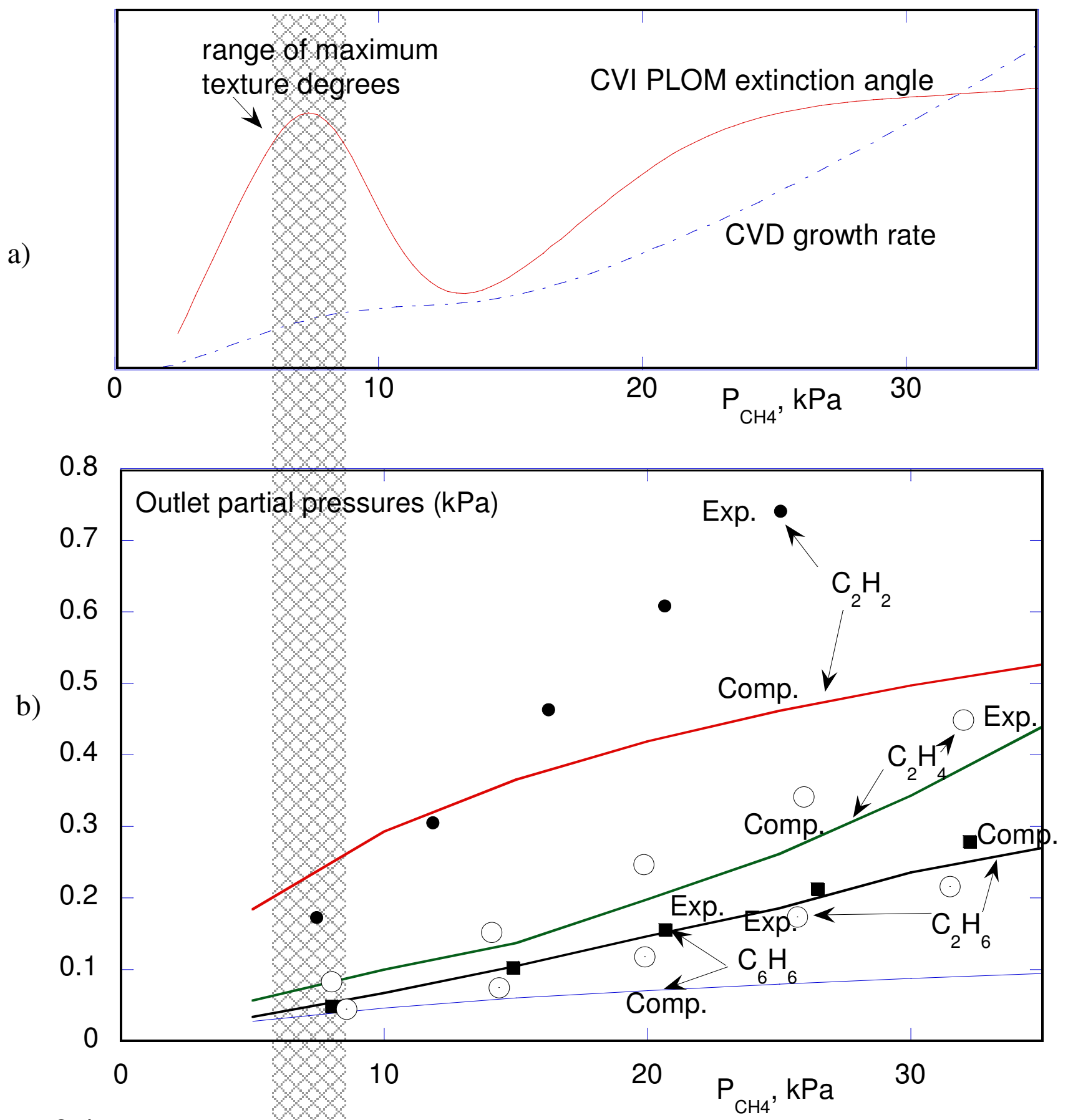

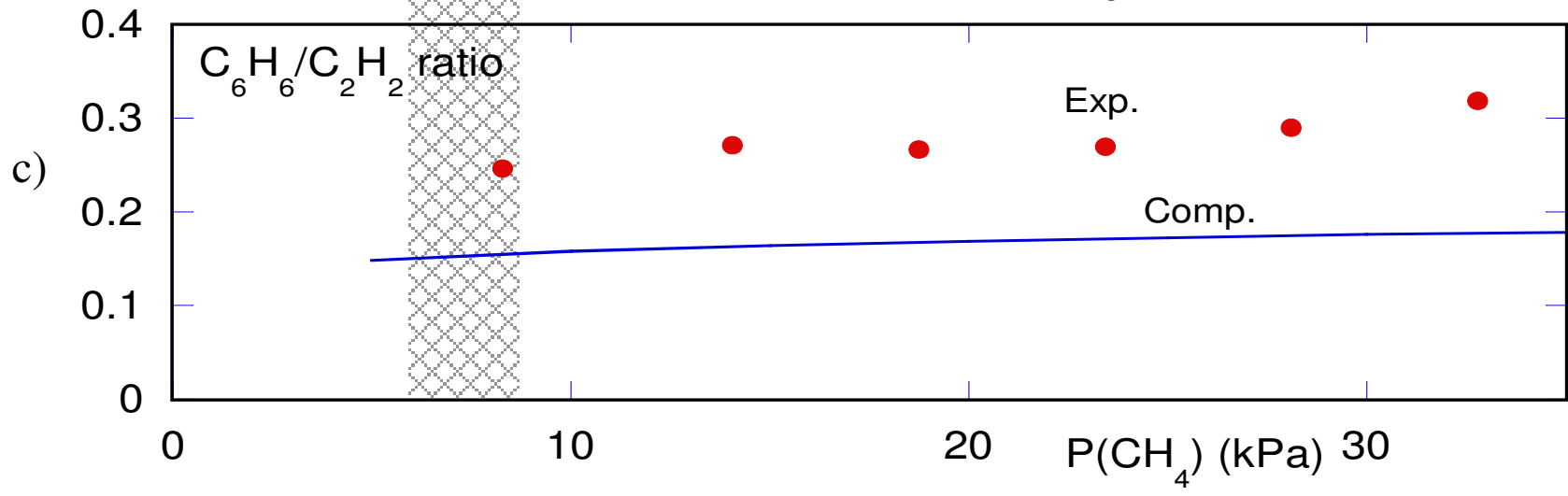

Fig. 3. 
Gerard L. Vignoles, Francis Langlais, Cédric Descamps, Arnaud Mouchon,

Hélène Le Poche, Nicolas Reuge and Nathalie Bertrand

Figure 3. 


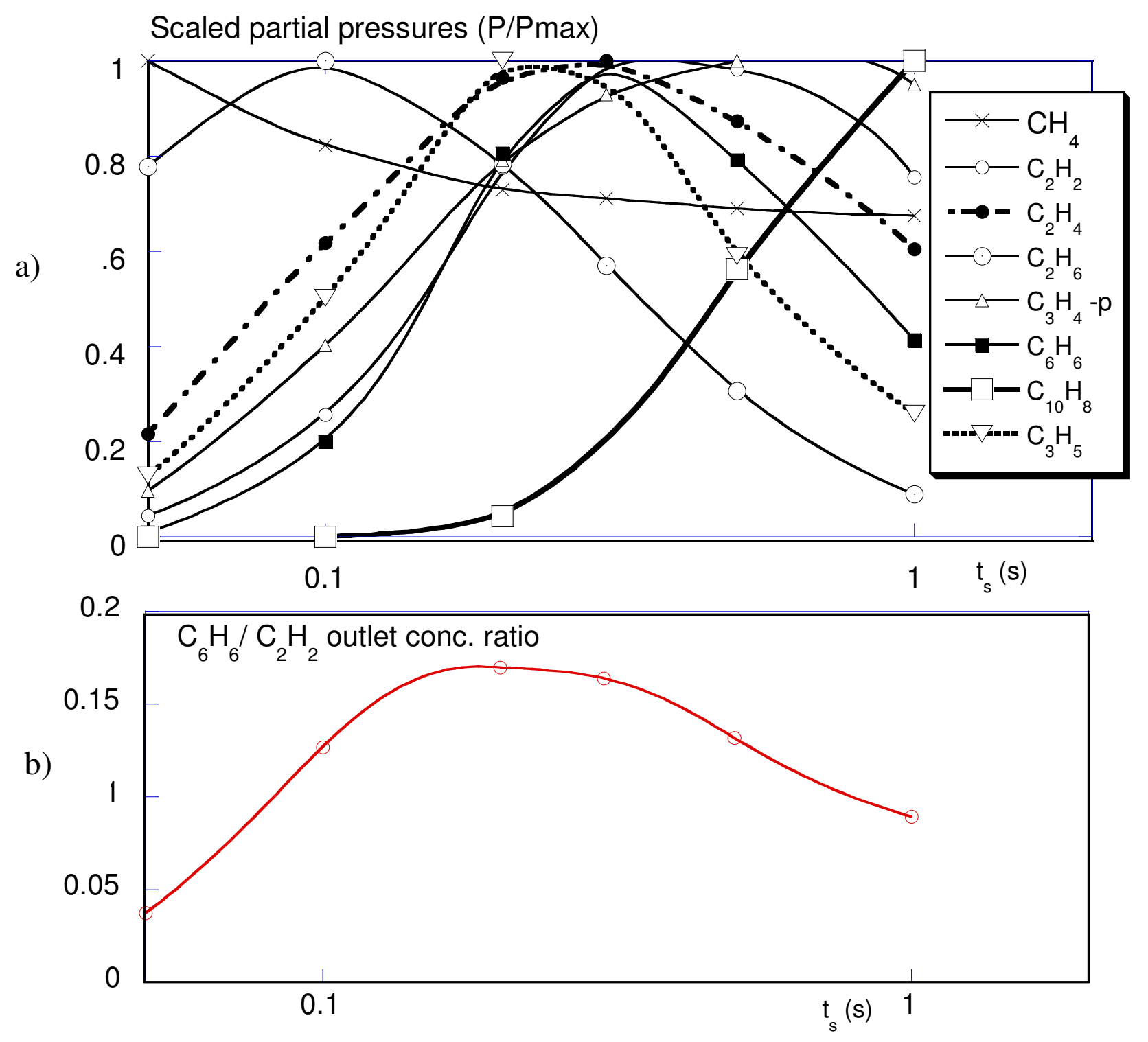

Fig. 4 
Gerard L. Vignoles, Francis Langlais, Cédric Descamps, Arnaud Mouchon,

Hélène Le Poche, Nicolas Reuge and Nathalie Bertrand

Figure 4. 


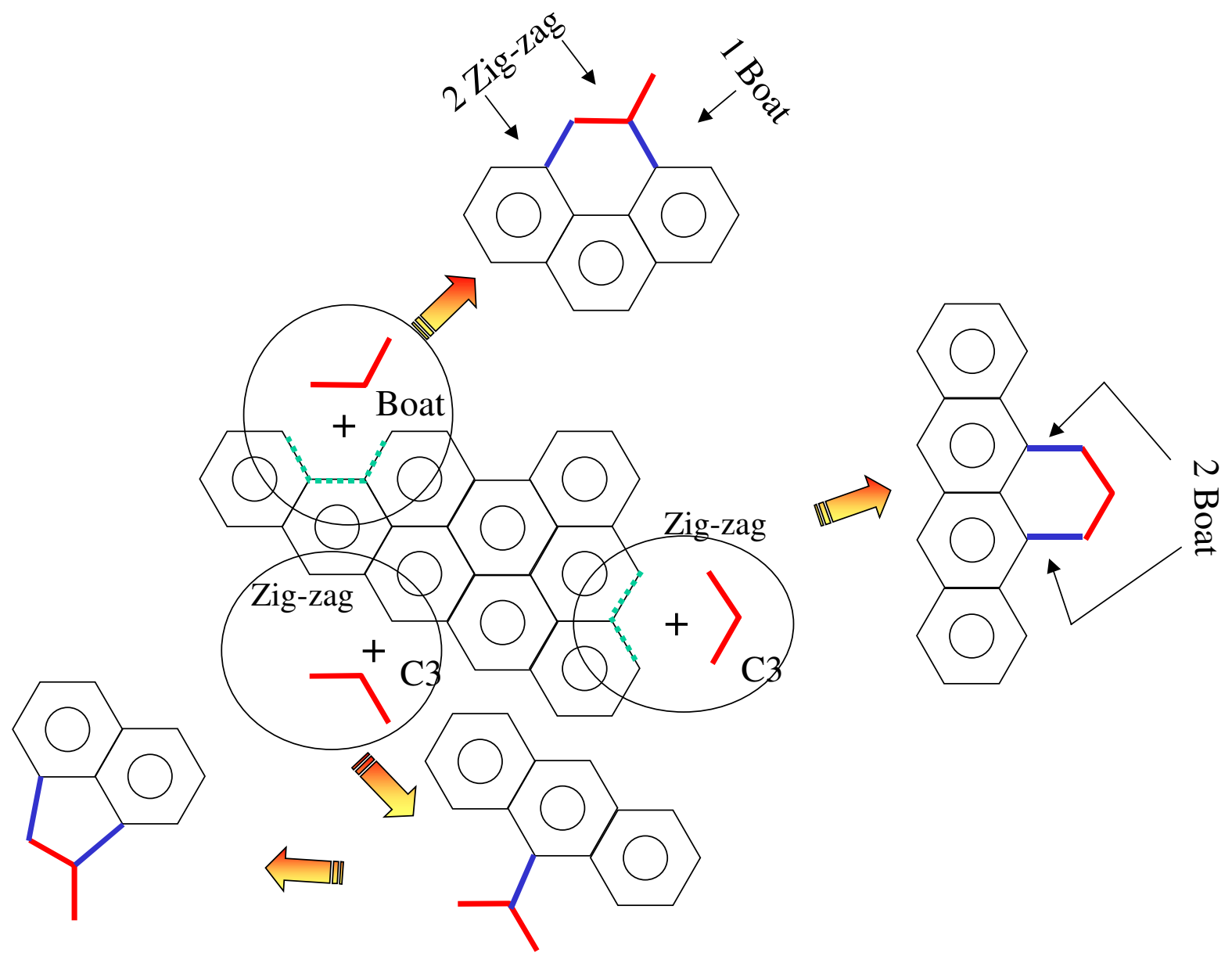

Fig. 5 
Gerard L. Vignoles, Francis Langlais, Cédric Descamps, Arnaud Mouchon,

Hélène Le Poche, Nicolas Reuge and Nathalie Bertrand

Figure 5 . 

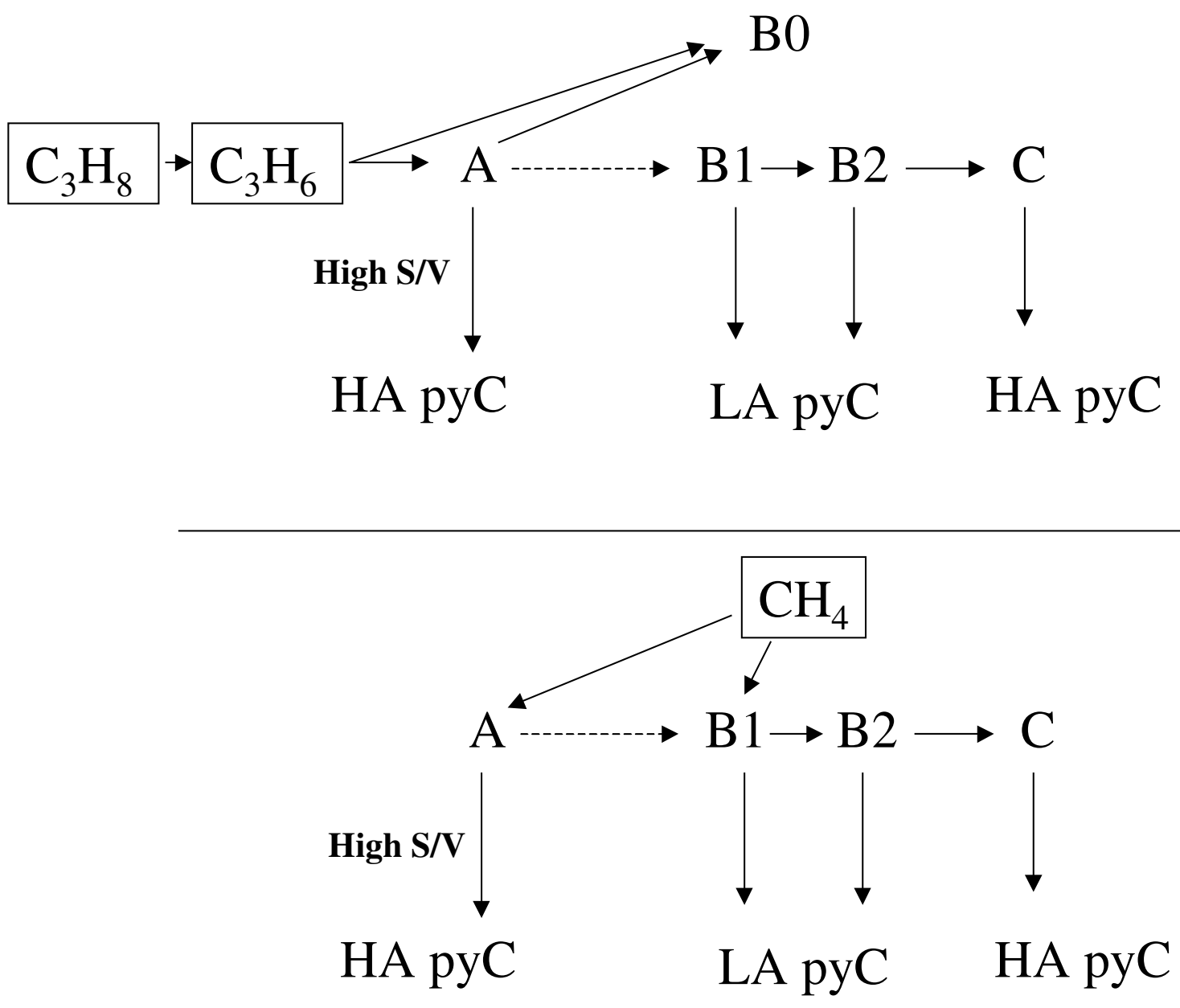

Fig. 6 
Gerard L. Vignoles, Francis Langlais, Cédric Descamps, Arnaud Mouchon,

Hélène Le Poche, Nicolas Reuge and Nathalie Bertrand

Figure 6. 